\title{
Daptomycin Resistance in Clinical MRSA Strains Is Associated with a High Biological Fitness Cost
}

\section{Melanie Roch ${ }^{1}$, Paula Gagetti1,2, James Davis ${ }^{3,4}$, Paola Ceriana ${ }^{2}$, Laura Errecalde ${ }^{5}$, Alejandra Corso ${ }^{2}$ and Adriana E. Rosato ${ }^{1 *}$}

\begin{abstract}
${ }^{1}$ Department of Pathology and Genomic Medicine, Center for Molecular and Translational Human Infectious Diseases Research, Houston Methodist Research Institute, Houston, TX, United States, ${ }^{2}$ Servicio Antimicrobianos, Instituto Nacional de Enfermedades Infecciosas, Administración Nacional de Laboratorios e Institutos de Salud, Buenos Aires, Argentina, ${ }^{3}$ Argonne National Laboratory (DOE), Lemont, IL, United States, ${ }^{4}$ Computation Institute, University of Chicago, Chicago, IL, United States, ${ }^{5}$ Departamento de Microbiologia, Hospital General de Agudos Juan Fernandez, Buenos Aires, Argentina
\end{abstract}

OPEN ACCESS

Edited by: Miklos Fuzi,

Semme/weis University, Hungary

Reviewed by:

Carla Novais,

Universidade do Porto, Portugal Antonio Cannatelli, University of Siena, Italy Liang-Yu Chen, Taipei Veterans General Hospital, Taiwan

${ }^{*}$ Correspondence: Adriana E. Rosato aerosato@houstonmethodist.org

Specialty section:

This article was submitted to Antimicrobials, Resistance

and Chemotherapy,

a section of the journal

Frontiers in Microbiology

Received: 11 September 2017 Accepted: 08 November 2017 Published: 05 December 2017

Citation:

Roch M, Gagetti P, Davis J, Ceriana P, Errecalde L, Corso A and

Rosato AE (2017) Daptomycin Resistance in Clinical MRSA Strains is Associated with a High Biological Fitness Cost.

Front. Microbiol. 8:2303. doi: 10.3389/fmicb.2017.02303
Daptomycin remains as one of the main treatment options for Methicillin-Resistant Staphylococcus aureus (MRSA). Sporadic resistance cases reported in patients treated with either daptomycin or glycopeptides are a growing concern. In a previous study, we described a clinical case of a patient with a community-acquired MRSA infection resistant to daptomycin and with intermediate resistance to vancomycin who developed a recurrent infection with a susceptible isogenic strain. In the present work, we further investigated the sequential events to determine whether the switch from a daptomycin resistance to a susceptible phenotype was due to a phenomenon of resistance reversion or recurrent infection with a susceptible strain. Pairwise competition experiments showed that the susceptible clinical recurrent SA6850 strain had increased fitness when compared to the resistant counterpart SA6820 strain. In fact, although we have demonstrated that reversion of daptomycin resistance to daptomycin susceptible can occur in vitro after serial passages in drug-free media, phylogenetic analysis suggested that the in vivo process was the result of a recurrent infection with a previous susceptible isolate carried by the patient rather than a resistance reversion of the strain. Whole genome sequence of evolved strains showed that daptomycin resistance in MRSA is associated with a high fitness cost mediated by mutations in mprF gene, revealed as a key element of the biological cost. Moreover, we determined that daptomycin resistance-associated fitness cost was independent of vancomycin intermediate resistance phenotype, as demonstrated in additional clinical MRSA vancomycin susceptible strains. This study highlights important observations as, despite daptomycin offers a useful treatment option for the patients with persistent infections, it has to be carefully monitored. The high fitness cost associated to daptomycin resistance may explain the reduced dissemination of daptomycin resistance and the absence of daptomycin reported outbreaks.

Keywords: MRSA, daptomycin, biological cost, in vivo 


\section{INTRODUCTION}

Staphylococcus aureus is one of the most common human pathogens isolated from both community-acquired (CA) and hospital-acquired (HA) infections. Antibiotic management of those infections has become complex over the last decades due to large spreads of multiresistant strains, notably methicillin resistant $S$. aureus (MRSA) and vancomycin intermediate S. aureus (VISA) (Stryjewski and Corey, 2014). Daptomycin (DAP), a calcium-dependent lipopeptide antibiotic with potent bactericidal activity (Kanafani and Corey, 2007), is the main alternative for the treatment of MRSA infections in cases of vancomycin-reduced susceptibility or vancomycin treatment failure (Liu et al., 2011). The mechanism of action involves the functional disruption of the cytoplasmic membrane which leads to its depolarization and cell death (Bayer et al., 2013). S. aureus strains with minimal inhibitory concentration $(\mathrm{MIC}) \leq 1 \mathrm{mg} / \mathrm{L}$ are classified as DAP susceptible (DAP-S) according to Clinical and Standard Laboratory Institute (CLSI, 2015). As no resistant breakpoint has been officially established, strains with MIC $>1 \mathrm{mg} / \mathrm{L}$ should be referred to as nonsusceptible. However, the term resistant (DAP-R) will be used in the present study to simplify the understanding.

Acquisition of DAP resistance in $S$. aureus is a stepwise and multifactorial process that includes cell membrane and probably cell wall perturbations (Baek et al., 2015; Tran et al., 2015). Mutations have been described in various genes including those associated with the cell membrane $(m p r F)$, cell wall (walKR, dltABCD, and $y y c G$ ), and RNA polymerase subunits ( $r p o C$ and $r p o B$ ), both in clinical isolates and laboratory derivatives (Friedman et al., 2006; Cui et al., 2010; Mehta et al., 2011; Mishra et al., 2014; Baek et al., 2015). Moreover, the twocomponent system VraSR, a key regulator of the cell wall synthesis, has also been shown to play an important role in DAP resistance regulation (Mehta et al., 2011). The gene most consistently associated with the resistance in clinical and in vitro mutant strains is $m p r F$, previously known as $f m t C$ (Peleg et al., 2012). The transmembrane protein MprF is responsible for the lysinylation of cell membrane phospholipids and their translocation to the outer leaflet of the cell membrane (Ernst et al., 2009). Gain-in-function mutations of $m p r F$ increase the cell membrane positive charges, and thus repulse the calcium-complexed DAP (Jones et al., 2008; Bayer et al., 2013).

Although the development of DAP resistance remains rare, cases have been reported in patients treated for high bacterial inoculum infections under antimicrobial therapies with glycopeptides or DAP, resulting in treatment failure, poor clinical outcomes, and high mortality (van Hal et al., 2011; Capone et al., 2016).

In a previous report, we described a clinical case of a patient in Argentina with a CA-MRSA infection with intermediate resistance to vancomycin (VISA) and resistance to DAP who developed a recurrence with a susceptible isogenic strain (Errecalde et al., 2013). A similar case was reported in 2016 by Capone et al. (2016) where under DAP treatment, a MRSA isolate acquired a heterogeneous DAP resistance that, surprisingly, came back to a susceptible phenotype when the treatment was switched. Both cases described a putative reversion of DAP resistance; however, the bases of this process remained to be determined.

In the present investigation, by using competition and phylogenetic analyses of the successive isolates originated from the same patient, we show that DAP resistance in MRSA is associated with a high fitness cost mediated by mutations in mprF gene, and that in vivo conversion of DAP resistance to a susceptible phenotype was in fact a recurrent infection rather than reversion.

\section{MATERIALS AND METHODS}

\section{Bacterial Strains}

Consecutive CA-MRSA clinical isolates (ST5-SCCmec type IVPVL+) were recovered from blood cultures from a patient with chronic kidney failure under dialysis with a history of fracture osteosynthesis: SA6819 (h-VISA/S-DAP), SA6820 (VISA/R-DAP), and SA6850 (VSSA/S-DAP) (Errecalde et al., 2013). The SA6820 $\Delta m p r F$ was obtained by transduction from the strain CK1001 using phage $80 \alpha$. The clinical isogenic pairs DAP-S and DAP-R CB-1631(S)/CB-1634(R) and CB5013(S)/CB-5014(R) (Mehta et al., 2011) described in Table 1 were used as representative strains displaying DAP-R and vancomycin susceptible phenotype (VSSA) for studies of DAP resistance fitness cost in vancomycin susceptible isolates.

\section{Antibiotics and Susceptibility Testing}

Daptomycin was provided by former Cubist/Merck Pharmaceuticals. Susceptibilities to vancomycin and DAP were performed by E-test (BioMérieux, Marcy l'Etoile, France). Antimicrobial susceptibility testing was performed by KirbyBauer method and according to the guidelines of the Clinical and Laboratory Standards with antimicrobial Sensi-disks (BD, Franklin Lakes, NJ, United States). The antimicrobial susceptible testing was used to complement genetic approaches to evaluate the antibiotic susceptible phenotype related to the occurrence of specific mutations and to follow up the identity of the strains during long-term passages experiments. The antibiotics tested were rifampicin (RIF), vancomycin (VAN), ciprofloxacin (CIP), and chloramphenicol (CM).

\section{Illumina Short Read Genome Sequencing}

Genomic DNA of all strains was extracted from an overnight culture grown in $\mathrm{BHI}$ at $37^{\circ} \mathrm{C}$ using the DNeasy Blood and Tissue Kit (QIAGEN, Hilden, Germany). Libraries were prepared from purified DNA using Nextera XT DNA Library Preparation Kit (Illumina, San Diego, CA, United States) and sequenced (50 nucleotide reads) with HiSeq 2000 instruments at the Epigenetics and Genomic Laboratory at Weill Cornell University, New York, NY, United States.

\section{Templated Genome Assembly}

Templated assemblies of the short reads were performed versus N315 (GenBank accession number BA000018) and the SNPs 
TABLE 1 | Description of the strains used in this study and their MICs obtained by $E$-test ${ }^{\circledR}$.

\begin{tabular}{|c|c|c|c|}
\hline Strain & MIC DAP (mg/L) & MIC VAN (mg/L) & Source and reference \\
\hline SA6819 & 0.25 & 2 & Clinical strain, Errecalde et al., 2013 \\
\hline SA6820 & 4 & 4 & Clinical strain, Errecalde et al., 2013 \\
\hline SA6850 & 0.25 & 1 & Clinical strain, Errecalde et al., 2013 \\
\hline SA6820-revertant D35 & 0.25 & 2 & Laboratory derived, this study \\
\hline SA6820 $\Delta m p r F$ & 0.75 & 3 & Laboratory derived, this study \\
\hline CB-1631 & 0.5 & 1 & Clinical strain, Cubist Pharmaceutical, Mehta et al., 2011 \\
\hline CB-1634 & 4 & 2 & Clinical strain, Cubist Pharmaceutical \\
\hline CB-5013 & 0.5 & 1 & Clinical strain, Cubist Pharmaceutical \\
\hline CB-5014 & 4 & 2 & Clinical strain, Cubist Pharmaceutical \\
\hline
\end{tabular}

identified using the Lasergene 13 Suite Software (DNASTAR, Inc., Madison, WI, United States).

\section{Sanger DNA Sequencing}

The mprF mutations were confirmed by PCR and Sanger sequencing using previously described mprF primers (Mehta et al., 2011) at the Nucleic Acid Research Facility at Genewiz (South Plainfield, NJ, United States).

\section{Phylogenetic Analysis}

All S. aureus strains of the ST5 MLST type were downloaded from PATRIC in April, 2017 (Wattam et al., 2017). Strains with a BioSample identifier that unambiguously linked to a single run accession were downloaded from the European Nucleotide Archive (Leinonen et al., 2011). When read files were paired-end, only one set in the pair was used for the comparison. Reads were aligned against N315 (PATRIC ID: 158879.11) and analyzed using the PATRIC variation service (Wattam et al., 2017), which uses BWA-MEM as the read aligner ${ }^{1}$ and FreeBayes as the SNP caller ${ }^{2}$. A SNP alignment was generated by using a method previously described (Leekitcharoenphon et al., 2012). A tree for the alignment was generated using FastTree. Genes for the RNA polymerase beta-prime subunit $(r p o C)$ were downloaded from PATRIC in April, 2017. Genes were aligned using MAFFT and a tree was built using FastTree. Trees were rendered using the iTOL website (Letunic and Bork, 2007).

\section{In Vitro DAP Resistance Reversion}

To access the ability of DAP resistance to revert, the resistant strain SA6820 was serially passaged daily in drug-free BHI broth. Every 5 days, enumeration of the DAP-resistant population was performed by plating the culture and its serial dilutions on BHI agar supplemented with calcium $\left(\mathrm{CaCl}_{2}, 50 \mathrm{mg} / \mathrm{L}\right)$ and containing or not DAP $2 \mathrm{mg} / \mathrm{L}$. The mutational genetic events of the resulting strain obtained after 35 days (SA6820revertant) were determined by whole genome sequencing and its fitness was compared against its parental SA6820 strain.

\footnotetext{
${ }^{1}$ https://arxiv.org/abs/1303.3997

${ }^{2}$ https://arxiv.org/abs/1207.3907
}

\section{Competition Experiments and Single Cultures}

Pairwise competition experiments between isogenic strains were conducted to compare their fitness (Andersson and Hughes, 2010). This method enables a good discrimination by taking into account the different component of the fitness, including the lag period and the exponential growth phase. Briefly, overnight cultures of the strains were mixed in equal proportion and inoculated at $10^{5} \mathrm{CFU} / \mathrm{mL}$ in $10 \mathrm{~mL}$ of BHI broth (BD, Franklin Lakes, NJ, United States). The mixed 24-h culture was then diluted 1:1000 in fresh BHI broth and repeated daily during 10 days. Enumeration of the DAP resistant population was performed every 2 days followed by plating the culture and its serial dilutions on BHI agar supplemented with calcium $\left(\mathrm{CaCl}_{2}\right.$, $50 \mathrm{mg} / \mathrm{L}$ ) and containing or not DAP $2 \mathrm{mg} / \mathrm{L}$. The final level of DAP resistance of the population was also evaluated after the 10 days of competition by E-test (BioMérieux, Marcy l'Etoile, France) with a $0.5 \mathrm{McF}$ inoculum and directly from the whole population cells. Single cultures of the resistant strain SA6820 were conducted in parallel of the competition assay by daily passage in drug-free BHI broth to assess their stability during the competition experiment. The susceptible strain SA6850 was also plated on BHI containing DAP to evaluate the spontaneous mutation frequency. For the competitive culture between SA6850 and SA6820 $\Delta m p r F$ that are both DAP-S, respectively; CIP $2 \mathrm{mg} / \mathrm{L}$ and CM $10 \mathrm{mg} / \mathrm{L}$ were used as antimicrobial markers to identify SA6850 (CIP-R) and for SA6820 $\Delta m p r F$ (CM-R).

\section{RESULTS AND DISCUSSION}

\section{mprF Is Associated with DAP Resistance in the ST5-SCCmec Type IV-PVL+ SA6820 Isolate}

The patient described in our previous study presented multiple comorbidities (diabetes, chronic kidney failure on hemodialysis, and hip fracture osteosynthesis), and was treated for a persistent bacteremia by various combination of antibiotics including VAN and DAP (van Hal et al., 2011; Bassetti et al., 2015; Figure 1). DAP resistance in SA6820 developed concomitantly with the acquisition of a full VISA resistance phenotype (isolate 
SA6820; VAN: $4 \mu \mathrm{g} / \mathrm{ml}$ ) after DAP treatment of a heterogeneousvancomycin intermediate resistant (h-VISA: $2 \mu \mathrm{g} / \mathrm{ml}$ ) isolate (SA6819) (Figure 1). Whole genome sequencing analysis revealed that SA6820 carried the L826F mprF mutation, one of the most commonly found in DAP resistant isolates (Bayer et al., 2015) and shown to be a major determinant of DAP resistance (Mehta et al., 2011; Yang et al., 2013). Additional SNPs in SA6820 were present in $r p o B$ gene at position $\mathrm{A} 477 \mathrm{D}$, and $v r a T$ at position H3_S7delinsP (Table 2). After treatment was switched to LNZ (linezolid), CIP and SXT (trimethoprim/sulfamethoxazole) and following a 3-month period of clinical improvement, the patient developed a recurrent infection due to a DAP susceptible isolate (SA6850).

However, sequence analysis of SA6850 displayed a mutation in the $r p o B$ gene at position $\mathrm{H} 481 \mathrm{Y}$ similar to the susceptible SA6819, while mutations in $m p r F$ and vraT were no longer present. The changes in genotype in SA6850 were consistent with its susceptible phenotype to DAP and VAN. The three isolates were shown to be classified as the same clone by PFGE and MLST (Errecalde et al., 2013) corresponding to ST5SCCmec type IV-PVL+, one of the main community-acquired clones circulating in Argentina; the high homology between these strains was confirmed by the phylogenetic tree based on rpoC (Supplementary Figure S1). These results were consistent with two hypotheses: (i) the susceptible SA6850 strain could be an in vivo revertant of the resistant SA6820 or (ii) it could be a recurrence of a previous version of the strain that the patient carried before acquisition of the DAP resistance. In both proposed hypotheses, the underlying condition was that the resistant SA6820 strain had a fitness disadvantage compared to
SA6850, which allowed the replacement of the resistant strain by a susceptible one.

\section{Strain SA6850 Outgrew SA6820 because of Its Better Fitness}

To test the fitness difference, we performed in vitro competitive cultures between DAP resistant SA6820 and its susceptible counterpart SA6850. After 10 days of subculture, the DAP susceptible strain SA6850 had completely outgrown the DAP resistant SA6820 (Figure 2A). MIC determination performed by $E$-test after 10 days of culture with inoculum from whole population (SA6820 + SA6850) revealed a susceptible phenotype with a DAP MIC at $0.38 \mu \mathrm{g} / \mathrm{mL}$. Residual population growing on BHI containing $2 \mathrm{mg} / \mathrm{L}$ DAP was shown to be spontaneous mutants of SA6850, as indicated by their characteristic resistance to CIP which was not carried by SA6820. In parallel, SA6820 resistant strain in single culture was shown to be stable during the 10 days of the experiment with a decrease of resistance of only $1 \log 10$ (Figure 2B) and an MIC by E-test of $3 \mu \mathrm{g} / \mathrm{mL}$. The results of in vitro competition experiments are in agreement with the observation that the DAP susceptible SA6850 strain had an increased fitness compared to its DAP resistant counterpart SA6820 strain.

\section{Investigation of the Origin of the DAP Susceptible SA6850}

To address our first hypothesis, i.e., whether DAP resistance may directly revert to a susceptible phenotype, we performed in vitro serial passages of SA6820 in drug-free BHI broth to determine

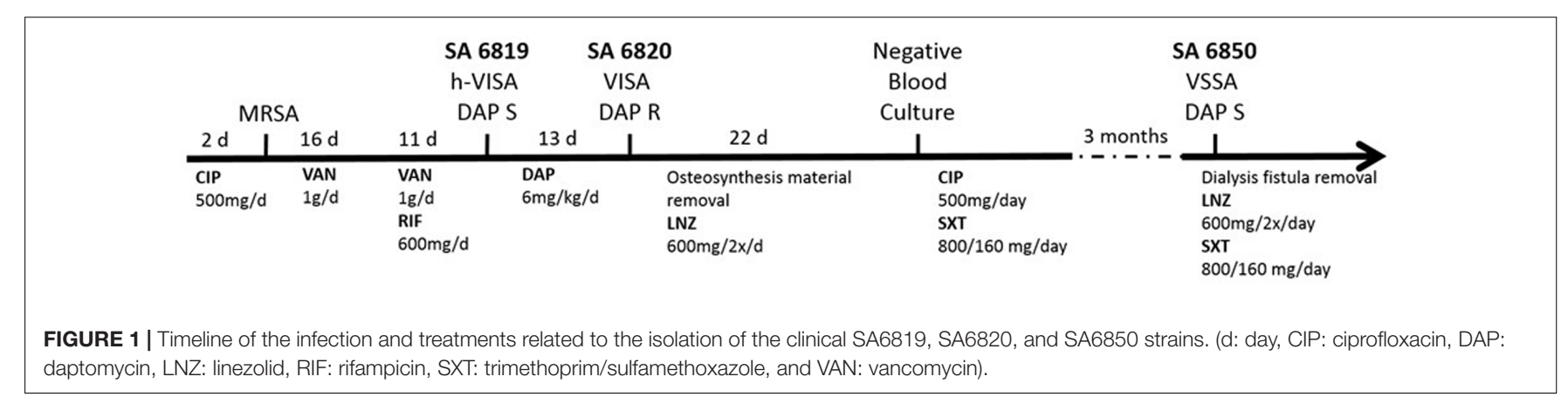

TABLE 2 | Most relevant mutations related to daptomycin and vancomycin resistance carried by the strains.

\begin{tabular}{|c|c|c|c|c|c|c|c|c|c|}
\hline Strain & rpoB & mprF & yycG (walk, vicK) & vras & vraT (yvqF /SA1702) & DAP & VAN & RIF & CIP \\
\hline SA6819 & H481Y & - & - & - & - & S & S & $\mathrm{R}$ & S \\
\hline SA6820 & A477D & L826F & - & - & H3_S7delinsP & $\mathrm{R}$ & I & $\mathrm{R}$ & S \\
\hline SA6850 & H481Y & - & - & - & - & S & S & $\mathrm{R}$ & $\mathrm{R}$ \\
\hline SA6820-revertant & A477D D471A & Y116fs* & - & E316V & H3_S7delinsP & $S$ & $S$ & $\mathrm{R}$ & $S$ \\
\hline SA6820 $\Delta m p r F$ & A477D & $\Delta m p r F$ & - & - & H3_S7delinsP & S & 1 & $\mathrm{R}$ & S \\
\hline CB-1631 & - & - & - & - & - & S & S & S & $R$ \\
\hline CB-1634 & - & L826F & K159fs & - & - & $\mathrm{R}$ & S & S & $\mathrm{R}$ \\
\hline CB-5013 & - & - & - & - & - & S & S & S & $\mathrm{R}$ \\
\hline CB-5014 & - & S337L & - & - & - & $\mathrm{R}$ & $S$ & $S$ & $\mathrm{R}$ \\
\hline
\end{tabular}

*The L826F mutation was still present but will not be translated due to the reading frameshift leading to an early stop codon in position 126. 
SA6820 (DAP-R) vs SA6850 (DAP-S)

A

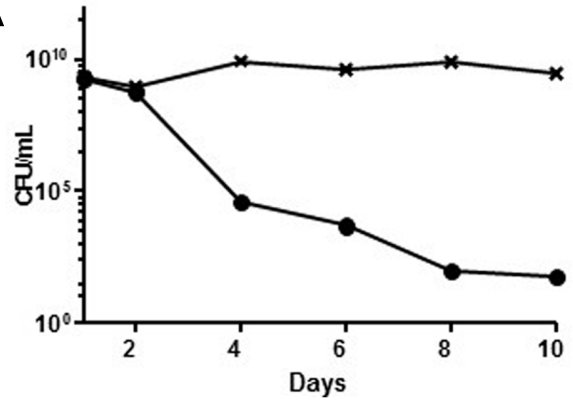

Single culture of SA6820 (DAP-R)

B

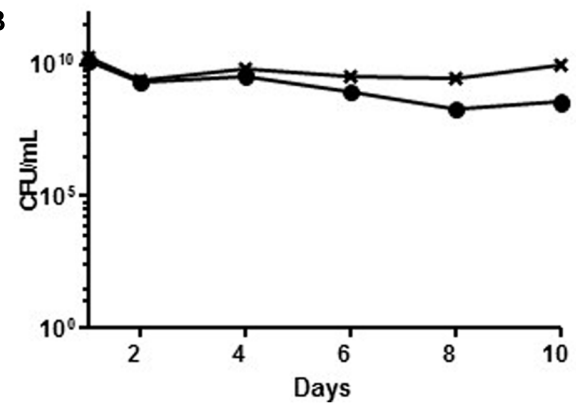

FIGURE 2 | (A) Competition experiment between the clinical SA6820 (DAP-R) and the clinical recurrent SA6850 (DAP-S) strains. The DAP-R sub-population of SA6820 is indicated with (•), the whole population (SA6820 + SA6850) growing on BHI agar without antibiotic is indicated with (x). (B) Single culture of SA6820 showed that in absence of competition with a susceptible strain, the resistance is stable during 10 days.

the ability of the DAP resistance to revert. We observed a progressive decrease of the DAP resistant population (Figure 3A) showing the slow reversion of the resistance. After 35 days, a fully susceptible strain with a DAP MIC at $0.5 \mu \mathrm{g} / \mathrm{mL}$, named SA6820-revertant, was recovered. A residual population growing on $\mathrm{BHI}+\mathrm{Ca}^{2+}$ containing $2 \mathrm{mg} / \mathrm{L}$ of DAP was present in similar proportion $\left(1.77 \times 10^{-7}\right)$ than the spontaneous DAP resistance frequency previously described in S. aureus (Kaatz et al., 2006). The SA6820-revertant also became susceptible to VAN, similarly to the clinical recurrent SA6850 strain.

To determine the in vitro generated gene polymorphisms associated with the conversion, the SA6820-revertant strain was full genome sequenced and compared to its parent SA-6820. Interestingly, the revertant strain showed a deletion of a single nucleotide $(\mathrm{T})$ in position 346 leading to a reading frameshift (Y116fs) and an early stop codon in position 126 of MprF, suggesting a direct role of $m p r F$ in the conversion process and thus in the fitness modification. Additional de novo mutations were also found in genes previously described to be associated with vancomycin intermediate resistance (Howden et al., 2010; Hafer et al., 2012) including D471A in RpoB and E316V VraS (Table 2), both of which may be also linked to its reversion.

Previous studies have shown that a resistant strain can rapidly acquire compensatory mutations to improve its fitness or adapt its metabolism to its culture media (Andersson and Hughes, 2010). However, in the strain SA6820, spontaneous inactivation of $\mathrm{MprF}$ itself occurred, resulting in the restoration of DAP susceptibility. This suggested that MprF inactivation improved the fitness of the strain which progressively outgrew the resistant population leading to a fully susceptible isolate. This result was supported by the competitive culture performed between the SA6820 and its in vitro revertant that showed a rapid decrease of the resistant population (Figure 3B). The difference of fitness was similar to the one observed between the clinical strains SA6820 and the in vivo DAP susceptible recurrent SA6850 strain (Figure 2B). Since VAN phenotype appears to be directly affected in vitro reversion of SA6820

A

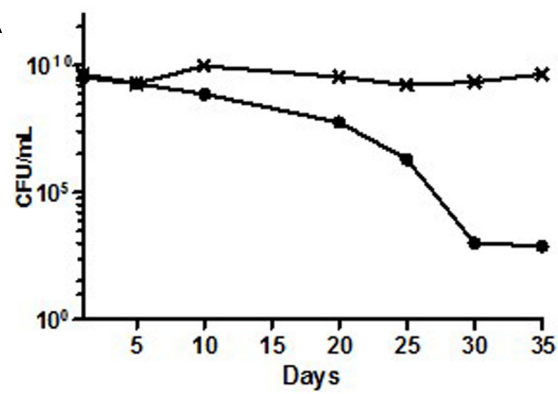

\section{SA6820 (DAP-R) vs SA6820-revertant (DAP-S)}

B

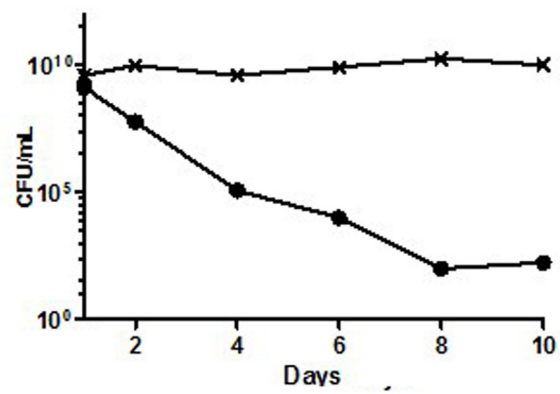

FIGURE 3 | (A) Progressive reversion of DAP resistance during extended in vitro single culture of the clinical DAP-R strain SA6820 by daily serial passages in drug-free BHI broth. Every 5 days, enumeration of the DAP resistant population was performed by plating the culture and its serial dilutions on BHI agar supplemented with calcium ( $\left.\mathrm{CaCl}_{2}, 50 \mathrm{mg} / \mathrm{L}\right)$ and containing or not DAP $2 \mathrm{mg} / \mathrm{L}$ resulting in (SA6820-revertant). The DAP-R sub-population of SA6820 is indicated with (•), the whole population (SA6820 + SA6820-revertant) growing on BHI agar without antibiotic is indicated with (x). (B) Competition experiment between the clinical strain SA6820 (DAP-R) and its in vitro revertant SA6820-revertant (DAP-S). The DAP-R sub-population of SA6820 is indicated with (•), the whole population growing on $\mathrm{BHI}$ agar without antibiotic is indicated with $(\mathbf{x})$. 


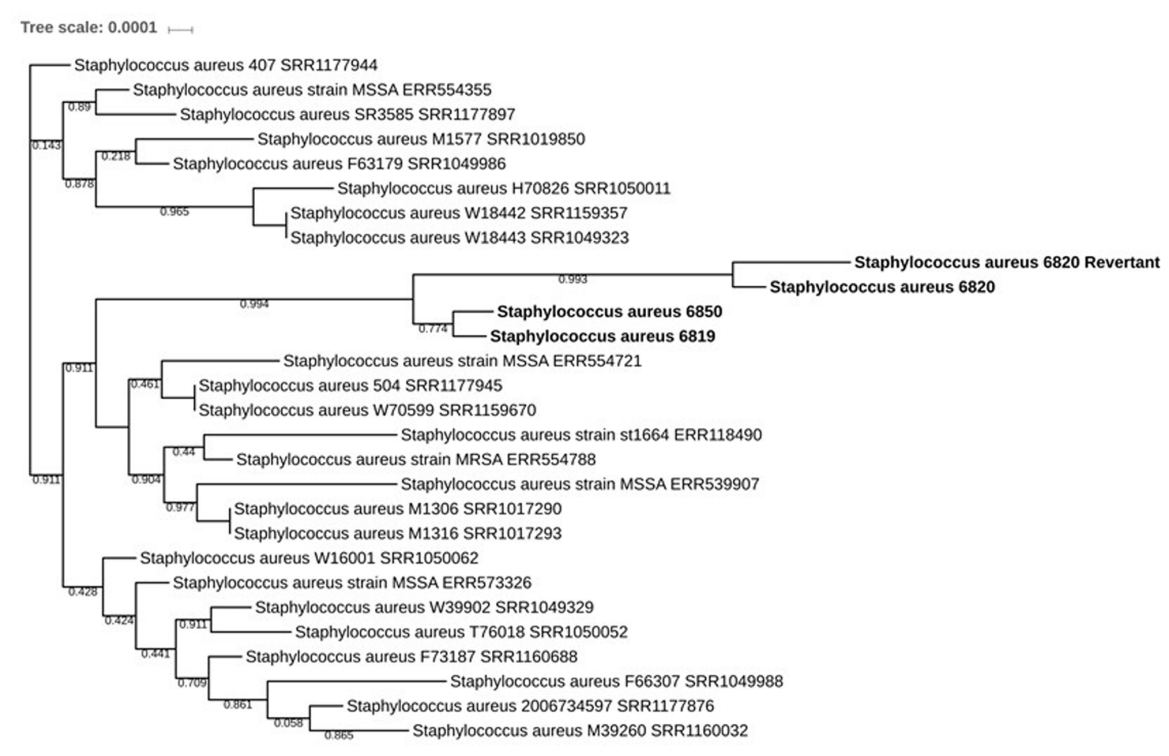

FIGURE 4 | Phylogenetic tree based on the SNP comparisons versus N315 showing the evolution of the different isolates: the strains SA6819, SA6820, SA6820-revertant, and SA6850 are shown in bold. All strains represented are from the ST5 MLST type.

during the process of reversion and may confound our results, the independent role of DAP resistance was confirmed in additional strains which were DAP resistant but VAN susceptible (these data are shown in separate section of this manuscript).

To investigate whether SA6850 was either an in vivo revertant or a recurrence of a susceptible strain previously carried by the patient, deeper analysis taking into account all the SNPs identified versus the $S$. aureus N315 reference strain was performed, with the goal of determining the proximity between SA6819, SA6820, SA6850, and the in vitro obtained SA6820-revertant strain. The phylogenetic tree revealed that the recurrent susceptible SA6850 strain was closer to the DAP susceptible h-VISA isolate SA6819 than to the VISA DAP resistant SA6820 (Figure 4). The high homology between these strains was confirmed by the phylogenetic tree based on rpoC (Supplementary Figure S1). These results suggested that the recurrent SA6850 strain was more likely to arise from a competition between a residual DAP susceptible population present in the patient and its environment than from a direct reversion of SA6820 strain. Furthermore, the reversion of DAP resistance to DAP susceptible phenotype observed in our patient was to some extent unexpected because there were no previous reports of cases describing a direct DAP resistance reversion. In fact, a similar clinical case has been then reported in where a heterogeneous MRSA DAP resistance isolate was selected under DAP treatment followed by a susceptible DAP phenotype strain when the antibiotic treatment was changed, however, the mechanistic bases of this phenotypic conversion were not further explored (Capone et al., 2016). Moreover, in a recent clinical MRSA strain pair obtained from a patient, it was demonstrated that DAP resistance reversion to a susceptible phenotype was obtained after DAP discontinuation, mechanistically associated with downregulation of cell wall associated genes (Iwata et al., 2017). These observations including ours, support the common notion that DAP resistant strains have the ability to revert to susceptible once DAP treatment is discontinued and switched to an alternative antimicrobial therapy.

\section{Impact of $m p r F$ Mutation on DAP Fitness}

The competition experiment between the SA6820 and SA6850 confirmed that the resistant strain had a lower fitness. This observation together with the spontaneous inactivation of MprF during serial passages of the resistant strain suggested that MprF, the major determinant of DAP resistance, may have a key role in the fitness cost of the resistance. To test this hypothesis, $m p r F$ was inactivated in the DAP resistant strain SA6820 by transduction of an insertional chloramphenicol cassette, resulting in the SA6820 $\Delta m p r F$ (CM-R) strain. As expected, this inactivation restored the DAP susceptibility and, of note, also decreased slightly the VAN intermediate resistance (Table 1). The $m p r F$ influence on VAN resistance had been previously suggested by different studies (Ruzin et al., 2003; Chen et al., 2015); however, the mechanism remains unknown. Competitive cultures performed between SA6820 $\Delta m p r F$ and SA6820 showed increased fitness of SA6820 $\Delta m p r F$ over SA6820 (Figure 5A), confirming the impact of $m p r F$ on the strain fitness. Competition between the two DAP susceptible strains SA6820 $\Delta m p r F$ and SA6850 showed no major difference of fitness; as shown in Figure 5B, after 10 days both remained in culture in similar proportion as confirmed by their resistance to both ciprofloxacin, characteristic of SA6850, and to chloramphenicol, marker of SA6820 $\Delta m p r F$.

In vitro studies proved the ability of DAP resistance to revert within a month (SA6820-revertant D35, DAP MIC: $0.25 \mu \mathrm{g} / \mathrm{mL}$ ), a mechanism mediated by the inactivation of $m p r F$, the key gene responsible for the resistance. This modification improved 
SA6820 (DAP-R) vs SA6820 $4 m p r F$ (DAP-S)

A

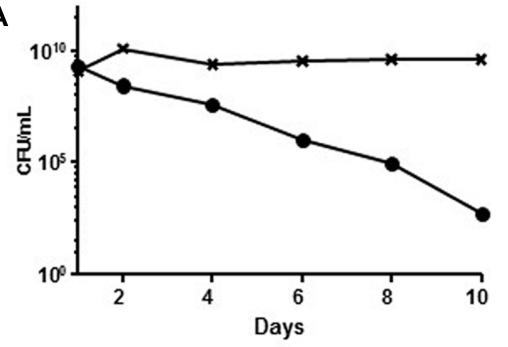

SA6850 (DAP-S) vs SA $6820 \Delta m p r F$ (DAP-S)

B

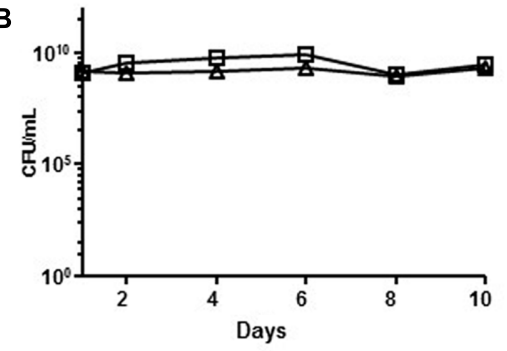

FIGURE 5 | (A) Competitive cultures performed between SA6820 $\Delta m p r F$ and SA6820 showed increased fitness of SA6820 $\Delta m p r F$ over SA6820 (DAP-R). DAP-R

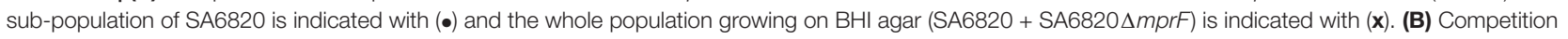
experiment between the two DAP-S strains: SA6850 (ciprofloxacin marker $\square$ ) and SA6820 $\Delta m p r F$ (chloramphenicol marker $\Delta$ ).

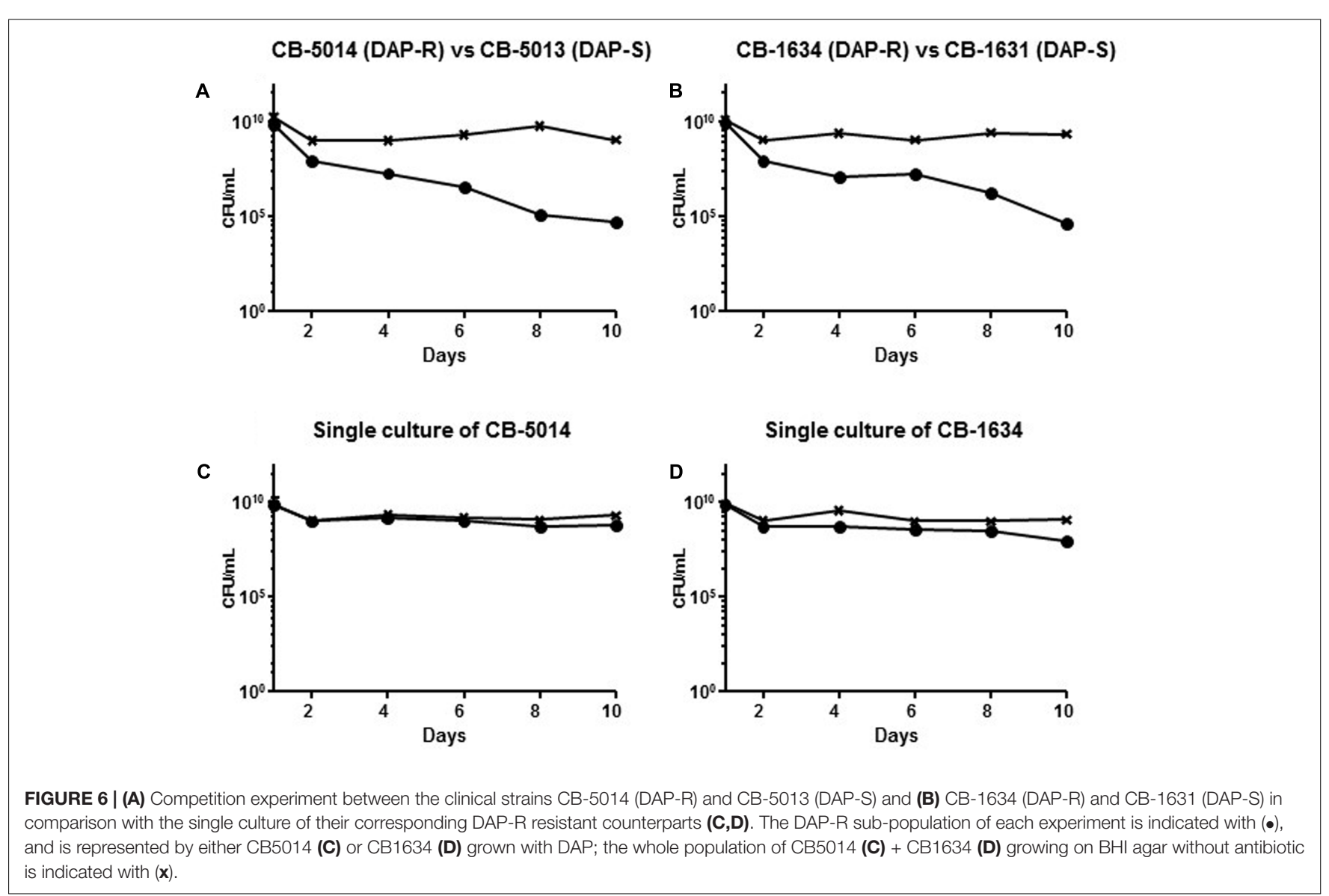

the fitness of $m p r F$-inactivated population which progressively outcompeted the resistant population. However, we cannot exclude the possibility that the in vivo process may differ from the in vitro one due to the potential complexity of its environment.

\section{DAP Resistance Fitness Cost in Vancomycin Susceptible Isolates}

The vancomycin intermediate resistance and rpoB SNPs carried by SA6820 have been previously associated with a high fitness cost (Boyle-Vavra et al., 2000; O’Neill et al., 2006; Noto et al., 2008), and could potentially confound our results as the strain SA6820 is a VISA strain, as most of the DAP resistant strains (Moise et al., 2009). To test the hypothesis that DAP resistance may have an independent fitness cost, we performed similar competition experiments (DAP susceptible versus resistant) using two pairs of VAN susceptible clinical isogenic strains, CB-5013/CB-5014 and CB-1631/CB-1634. These strains carried different $m p r F$ mutations, i.e., L826F in the hydrophobic N-terminal translocase, strain CB-1634, and S337L 
in the central bifunctional domain, strain CB-5014 (Ernst et al., 2009; Bayer et al., 2015). By performing competition analysis, we found that DAP susceptible strains, i.e., CB-5013 and CB-1631, displayed a better fitness as they both outcompeted their corresponding isogenic resistant counterparts (Figure 6). These results strengthened our hypothesis showing the mprFmediated DAP resistance fitness cost, and demonstrated that it was independent of the presence of $r p o B$ mutations and VAN intermediate resistance.

\section{CONCLUSION}

In summary, our study demonstrates for the first time the fitness cost of DAP resistance in clinical $S$. aureus and the role of $m p r F$ in this phenomenon. Therefore, due to the loss of fitness, DAP resistance conversion to DAP susceptibility can occur in patients when antibiotic pressure is removed. Our study demonstrate the possibility that a patient can carry strains with different resistance phenotypes, which can compete with each other and have different selective advantage in different contexts (presence or absence of antibiotics) in this particular case DAP. In fact, although we have demonstrated that reversion of DAP-R to DAP-S can occur in vitro, our results revealed that that in vivo conversion of DAP resistance to a susceptible phenotype was in fact a recurrent infection rather than reversion as also observed in additional reported cases (Capone et al., 2016; Iwata et al., 2017)

These findings are in support of one of our initial hypotheses in where the resistant SA6820 strain had a fitness disadvantage compared to SA6850, therefore favoring the replacement of the resistant strain by a susceptible one. In fact, MICs should be carefully monitored during the course of persistent infections or recurrence in where DAP may offer a useful treatment option for the patient (Capone et al., 2016), although the probability of resistance re-selection when the antibiotic treatment is re-introduced remains unknown. The difficulty to sustain DAP

\section{REFERENCES}

Andersson, D. I., and Hughes, D. (2010). Antibiotic resistance and its cost: is it possible to reverse resistance? Nat. Rev. Microbiol. 8, 260-271. doi: 10.1038/ nrmicro2319

Baek, K. T., Thogersen, L., Mogenssen, R. G., Mellergaard, M., Thomsen, L. E., Petersen, A., et al. (2015). Stepwise decrease in daptomycin susceptibility in clinical Staphylococcus aureus isolates associated with an initial mutation in rpoB and a compensatory inactivation of the clpX gene. Antimicrob. Agents Chemother. 59, 6983-6991. doi: 10.1128/AAC.01303-15

Bassetti, M., Villa, G., Ansaldi, F., De Florentiis, D., Tascini, C., Cojutti, P., et al. (2015). Risk factors associated with the onset of daptomycin non-susceptibility in Staphylococcus aureus infections in critically ill patients. Intensive Care Med. 41, 366-368. doi: 10.1007/s00134-014-3571-6

Bayer, A. S., Mishra, N. N., Chen, L., Kreiswirth, B. N., Rubio, A., and Yang, S. J. (2015). Frequency and distribution of single-nucleotide polymorphisms within mprF in methicillin-resistant Staphylococcus aureus clinical isolates and their role in cross-resistance to daptomycin and host defense antimicrobial peptides. Antimicrob. Agents Chemother. 59, 4930-4937. doi: 10.1128/AAC.00 970-15

Bayer, A. S., Schneider, T., and Sahl, H. G. (2013). Mechanisms of daptomycin resistance in Staphylococcus aureus: role of the cell membrane and cell resistance due to high fitness cost may explain the reduced dissemination of DAP resistance as well as the absence of DAP reported outbreaks. However, our study does not rule out that DAP transmission may occur in the presence of high antibiotic pressure, such as that existing in an intensive care unit where small outbreaks of vancomycin intermediate resistance have been previously reported (Pina et al., 2000; de Lassence et al., 2006).

\section{AUTHOR CONTRIBUTIONS}

MR: conceived and designed experiments. JD: performed genome and phylogeny analysis. PG: performed data analysis. MR, PG, and AR: drafted the manuscript. LE, PC, and AC: provided clinical strains and performed data analysis. AR, MR, and JD edited the manuscript and did final preparation.

\section{FUNDING}

This work was supported by NIH-1R56AI102503-01A1 (AR).

\section{ACKNOWLEDGMENT}

We acknowledge the Epigenomics Core Facility at Weill Cornell Medicine (New York, NY, United States) for their resources and assistance with whole genome sequencing experiments.

\section{SUPPLEMENTARY MATERIAL}

The Supplementary Material for this article can be found online at: https://www.frontiersin.org/articles/10.3389/fmicb. 2017.02303/full\#supplementary-material

wall. Ann. N. Y. Acad. Sci. 1277, 139-158. doi: 10.1111/j.1749-6632.2012. 06819.x

Boyle-Vavra, S., Berke, S. K., Lee, J. C., and Daum, R. S. (2000). Reversion of the glycopeptide resistance phenotype in Staphylococcus aureus clinical isolates. Antimicrob. Agents Chemother. 44, 272-277.

Capone, A., Cafiso, V., Campanile, F., Parisi, G., Mariani, B., Petrosillo, N., et al. (2016). In vivo development of daptomycin resistance in vancomycinsusceptible methicillin-resistant Staphylococcus aureus severe infections previously treated with glycopeptides. Eur. J. Clin. Microbiol. Infect. Dis. 35, 625-631. doi: 10.1007/s10096-016-2581-4

Chen, C. J., Huang, Y. C., and Chiu, C. H. (2015). Multiple pathways of crossresistance to glycopeptides and daptomycin in persistent MRSA bacteraemia. J. Antimicrob. Chemother. 70, 2965-2972. doi: 10.1093/jac/dkv225

CLSI (2015). Clinical and Laboratory Standards Institute, M100-S25, Performance Standards for Antimicrobial Susceptibility Testing, 25th Informational Supplement. Wayne, PA: CLSI.

Cui, L., Isii, T., Fukuda, M., Ochiai, T., Neoh, H. M., Camargo, I. L., et al. (2010). An RpoB mutation confers dual heteroresistance to daptomycin and vancomycin in Staphylococcus aureus. Antimicrob. Agents Chemother. 54, 5222-5233. doi: 10.1128/AAC.00437-10

de Lassence, A., Hidri, N., Timsit, J. F., Joly-Guillou, M. L., Thiery, G., Boyer, A., et al. (2006). Control and outcome of a large outbreak of colonization and 
infection with glycopeptide-intermediate Staphylococcus aureus in an intensive care unit. Clin. Infect. Dis 42, 170-178. doi: 10.1086/498898

Ernst, C. M., Staubitz, P., Mishra, N. N., Yang, S. J., Hornig, G., Kalbacher, H., et al. (2009). The bacterial defensin resistance protein MprF consists of separable domains for lipid lysinylation and antimicrobial peptide repulsion. PLOS Pathog. 5:e1000660. doi: 10.1371/journal.ppat.1000660

Errecalde, L., Ceriana, P., Gagetti, P., Erbin, M., Duarte, A., Cuatz, D., et al. (2013). [First isolation in Argentina of community-acquired methicillinresistant Staphylococcus aureus with intermediate susceptibility to vancomycin and nonsusceptibility to daptomycin]. Rev. Argent Microbiol. 45, 99-103.

Friedman, L., Alder, J. D., and Silverman, J. A. (2006). Genetic changes that correlate with reduced susceptibility to daptomycin in Staphylococcus aureus. Antimicrob. Agents Chemother. 50, 2137-2145.

Hafer, C., Lin, Y., Kornblum, J., Lowy, F. D., and Uhlemann, A. C. (2012). Contribution of selected gene mutations to resistance in clinical isolates of vancomycin-intermediate Staphylococcus aureus. Antimicrob. Agents Chemother. 56, 5845-5851. doi: 10.1128/AAC.01139-12

Howden, B. P., Davies, J. K., Johnson, P. D., Stinear, T. P., and Grayson, M. L. (2010). Reduced vancomycin susceptibility in Staphylococcus aureus, including vancomycin-intermediate and heterogeneous vancomycin-intermediate strains: resistance mechanisms, laboratory detection, and clinical implications. Clin. Microbiol. Rev. 23, 99-139. doi: 10.1128/CMR.00042-09

Iwata, Y., Satou, K., Tsuzuku, H., Furuichi, K., Senda, Y., Sakai-Takemori, Y., et al. (2017). Down-regulation of the two-component system and cellwall biosynthesis-related genes was associated with the reversion to daptomycin susceptibility in daptomycin non-susceptible methicillin-resistant Staphylococcus aureus. Eur. J. Clin. Microbiol. Infect. Dis. doi: 10.1007/s10096017-2999-3 [Epub ahead of print].

Jones, T., Yeaman, M. R., Sakoulas, G., Yang, S. J., Proctor, R. A., Sahl, H. G., et al. (2008). Failures in clinical treatment of Staphylococcus aureus infection with daptomycin are associated with alterations in surface charge, membrane phospholipid asymmetry, and drug binding. Antimicrob. Agents Chemother. 52, 269-278.

Kaatz, G. W., Lundstrom, T. S., and Seo, S. M. (2006). Mechanisms of daptomycin resistance in Staphylococcus aureus. Int. J. Antimicrob. Agents 28, 280-287. doi: 10.1016/j.ijantimicag.2006.05.030

Kanafani, Z. A., and Corey, G. R. (2007). Daptomycin: a rapidly bactericidal lipopeptide for the treatment of Gram-positive infections. Expert Rev. Anti Infect. Ther. 5, 177-184. doi: 10.1586/14787210.5.2.177

Leekitcharoenphon, P., Kaas, R. S., Thomsen, M. C., Friis, C., Rasmussen, S., and Aarestrup, F. M. (2012). snpTree-a web-server to identify and construct SNP trees from whole genome sequence data. BMC Genomics 13(Suppl. 7):S6. doi: 10.1186/1471-2164-13-S7-S6

Leinonen, R., Akhtar, R., Birney, E., Bower, L., Cerdeno-Tarraga, A., Cheng, Y., et al. (2011). The European nucleotide archive. Nucleic Acids Res. 39, D28-D31. doi: $10.1093 / \mathrm{nar} / \mathrm{gkq} 967$

Letunic, I., and Bork, P. (2007). Interactive tree of life (iTOL): an online tool for phylogenetic tree display and annotation. Bioinformatics 23, 127-128. doi: 10.1093/bioinformatics/btl529

Liu, C., Bayer, A., Cosgrove, S. E., Daum, R. S., Fridkin, S. K., Gorwitz, R. J., et al. (2011). Clinical practice guidelines by the infectious diseases society of America for the treatment of methicillin-resistant Staphylococcus aureus infections in adults and children: executive summary. Clin. Infect. Dis. 52, 285-292. doi: $10.1093 / \mathrm{cid} / \mathrm{cir} 034$

Mehta, S., Cuirolo, A. X., Plata, K. B., Riosa, S., Silverman, J. A., Rubio, A., et al. (2011). VraSR two-component regulatory system contributes to mprFmediated decreased susceptibility to daptomycin in-vivo-selected MRSA clinical strains. Antimicrob. Agents Chemother. 56, 92-102. doi: 10.1128/AAC. 00432- 10
Mishra, N. N., Bayer, A. S., Weidenmaier, C., Grau, T., Wanner, S., Stefani, S., et al. (2014). Phenotypic and genotypic characterization of daptomycin-resistant methicillin-resistant Staphylococcus aureus strains: relative roles of $\mathrm{mprF}$ and dlt operons. PLOS ONE 9:e107426. doi: 10.1371/journal.pone.0107426

Moise, P. A., North, D., Steenbergen, J. N., and Sakoulas, G. (2009). Susceptibility relationship between vancomycin and daptomycin in Staphylococcus aureus: facts and assumptions. Lancet Infect. Dis. 9, 617-624. doi: 10.1016/S14733099(09)70200-2

Noto, M. J., Fox, P. M., and Archer, G. L. (2008). Spontaneous deletion of the methicillin resistance determinant, mecA, partially compensates for the fitness cost associated with high-level vancomycin resistance in Staphylococcus aureus. Antimicrob. Agents Chemother. 52, 1221-1229. doi: 10.1128/AAC.01164-07

O’Neill, A. J., Huovinen, T., Fishwick, C. W., and Chopra, I. (2006). Molecular genetic and structural modeling studies of Staphylococcus aureus RNA polymerase and the fitness of rifampin resistance genotypes in relation to clinical prevalence. Antimicrob. Agents Chemother. 50, 298-309. doi: 10.1128/ AAC.50.1.298-309.2006

Peleg, A. Y., Miyakis, S., Ward, D. V., Earl, A. M., Rubio, A., Cameron, D. R., et al. (2012). Whole genome characterization of the mechanisms of daptomycin resistance in clinical and laboratory derived isolates of Staphylococcus aureus. PLOS ONE 7:e28316. doi: 10.1371/journal.pone.0028316

Pina, P., Marliere, C., Vandenesch, F., Bedos, J. P., Etienne, J., and Allouch, P. Y. (2000). An outbreak of Staphylococcus aureus strains with reduced susceptibility to glycopeptides in a French general hospital. Clin. Infect. Dis. 31, 1306-1308. doi: $10.1086 / 317464$

Ruzin, A., Severin, A., Moghazeh, S. L., Etienne, J., Bradford, P. A., Projan, S. J., et al. (2003). Inactivation of mprF affects vancomycin susceptibility in Staphylococcus aureus. Biochim. Biophys. Acta 1621, 117-121.

Stryjewski, M. E., and Corey, G. R. (2014). Methicillin-resistant Staphylococcus aureus: an evolving pathogen. Clin. Infect. Dis. 58(Suppl. 1), S10-S19. doi: 10.1093/cid/cit613

Tran, T. T., Munita, J. M., and Arias, C. A. (2015). Mechanisms of drug resistance: daptomycin resistance. Ann. N. Y. Acad. Sci. 1354, 32-53. doi: 10.1111/nyas. 12948

van Hal, S. J., Paterson, D. L., and Gosbell, I. B. (2011). Emergence of daptomycin resistance following vancomycin-unresponsive Staphylococcus aureus bacteraemia in a daptomycin-naive patient-a review of the literature. Eur. J. Clin. Microbiol. Infect. Dis. 30, 603-610. doi: 10.1007/s10096-010$1128-3$

Wattam, A. R., Davis, J. J., Assaf, R., Boisvert, S., Brettin, T., Bun, C., et al. (2017). Improvements to PATRIC, the all-bacterial bioinformatics database and analysis resource center. Nucleic Acids Res. 45, D535-D542. doi: 10.1093/nar/ gkw1017

Yang, S. J., Mishra, N. N., Rubio, A., and Bayer, A. S. (2013). Causal role of single nucleotide polymorphisms within the mprF gene of Staphylococcus aureus in daptomycin resistance. Antimicrob. Agents Chemother. 57, 5658-5664. doi: 10.1128/AAC.01184-13

Conflict of Interest Statement: The authors declare that the research was conducted in the absence of any commercial or financial relationships that could be construed as a potential conflict of interest.

Copyright $\odot 2017$ Roch, Gagetti, Davis, Ceriana, Errecalde, Corso and Rosato. This is an open-access article distributed under the terms of the Creative Commons Attribution License (CC BY). The use, distribution or reproduction in other forums is permitted, provided the original author(s) or licensor are credited and that the original publication in this journal is cited, in accordance with accepted academic practice. No use, distribution or reproduction is permitted which does not comply with these terms. 